\title{
$O$ relacionamento entre pessoas e serpentes no leste de Minas Gerais, sudeste do Brasil
}

\author{
Mário Ribeiro de Moura ${ }^{1,3}$, Henrique Caldeira Costa ${ }^{1}$, Vinícius de Avelar São-Pedro², \\ Vitor Dias Fernandes ${ }^{1}$ \& Renato Neves Feio ${ }^{I}$
}

\author{
${ }^{1}$ Museu de Zoologia João Moojen, Universidade Federal de Viçosa - UFV, \\ Vila Gianetti, 32, CEP 36570-000, Viçosa, MG, Brasil \\ ${ }^{2}$ Laboratório de Ecologia Evolutiva de Anfíbios e Répteis, \\ Universidade Federal do Rio Grande do Norte - UFRN, CEP 59072-970, Natal, RN, Brasil \\ ${ }^{3}$ Autor para correspondência: Mário Ribeiro de Moura, e-mail: mariormoura@gmail.com
}

MOURA, M.R., COSTA, H.C., SÃO-PEDRO, V.A., FERNANDES, V.D. \& FEIO, R.N. The relationship between people and snakes in eastern Minas Gerais, southeastern Brazil. Biota Neotrop. 10(4): http://www. biotaneotropica.org.br/v10n4/en/abstract?article+bn02410042010.

\begin{abstract}
The popular knowledge about snakes, including the practices adopted in cases of snakebite, was analysed in this ethnozoological study performed in Araponga region and vicinities of Serra do Brigadeiro (Brigadeiro Mountain Range), Atlantic Forest of Minas Gerais state, southeastern Brazil. Between August and November 2008, interviews were conducted with 50 residents of rural areas of Araponga, and 20 employees of the Serra do Brigadeiro State Park (PESB). In relation to social and cultural profile, these two groups differed only on the level of education (higher among the park staff), with the same distributions for age and religion. There was also a lower level of education among older individuals, a possible reflection of improvements in the social conditions in that region, which would have provided greater access to schools in recent decades. In general, both groups demonstrated adequate knowledge about prevention and procedures in cases of snakebite ( $78.2 \%$ reported seeking medical attention in case of snakebite). The use of folk medicine for treatment of snakebite proved to be a practice falling into disuse, reported by approximately $21 \%$ of respondents. Most respondents $(57.14 \%$ ) said they did not know the difference between a poisonous and a non-poisonous snake, and $66.67 \%$ showed adequate knowledge of the season when snake encounters are more likely to happen. The "Araponga" group was more hostile concerning to possible encounters with snakes, with $43 \%$ of people saying they would kill the animal, against $5 \%$ in the "PESB" group. The educational level of the respondents was decisive in determining the kind of attitude taken against snakes, and those with higher levels of education showed to be the less hostile ones. People with lower educational levels were more likely to consider all snakes as dangerous, and they also proved to be more hostile to these animals. More contact with scientific and environmental education activities seems to have been decisive for the higher tolerance to snakes by the "PESB" group. The implementation of activities of environmental education for the population of Araponga can increase the awareness of the importance of snakes, instructing those who still consider them intrinsically harmful.
\end{abstract}

Keywords: ethnozoology, education, Serpentes, Serra do Brigadeiro, rural communities.

MOURA, M.R., COSTA, H.C., SÃO-PEDRO, V.A., FERNANDES, V.D. \& FEIO, R.N. O relacionamento entre pessoas e serpentes no leste de Minas Gerais, sudeste do Brasil. Biota Neotrop. 10(4): http://www. biotaneotropica.org.br/v10n4/pt/abstract?article+bn02410042010.

Resumo: O conhecimento popular sobre as serpentes, incluindo as práticas adotadas em casos de acidentes ofídicos, foi abordado neste estudo etnozoológico realizado na região de Araponga e entorno da Serra do Brigadeiro, Mata Atlântica do Estado de Minas Gerais, região Sudeste do Brasil. Entre agosto e novembro de 2008 foram realizadas entrevistas com 50 moradores da zona rural de Araponga e 20 funcionários do Parque Estadual da Serra do Brigadeiro (PESB). Em relação ao perfil cultural e social, os grupos avaliados diferiram entre si somente quanto ao nível de escolaridade (maior entre os funcionários do parque), apresentando as mesmas distribuições em relação a idade e religião. Também se verificou menor nível de escolaridade entre os indivíduos mais velhos. Em geral, ambos os grupos de entrevistados demonstraram conhecimento adequado sobre prevenção e procedimentos em casos de acidente ofídico ( $78,2 \%$ afirmaram procurar atendimento médico em caso de ofidismo). A utilização de substâncias da medicina popular para tratamento de acidentes ofídicos mostrou-se uma prática em desuso, relatada por aproximadamente $21 \%$ dos entrevistados. A maioria dos entrevistados $(57,14 \%)$ afirmou não saber diferenciar uma serpente peçonhenta de uma não peçonhenta, e $66,67 \%$ demonstraram reconhecer o período chuvoso como aquele com maior frequência no encontro com serpentes. O grupo "Araponga" mostrou-se mais hostil em relação a encontros com serpentes, com $43 \%$ dos indivíduos afirmando matarem o animal, contra apenas 5\% do grupo "PESB". A escolaridade do entrevistado foi decisiva no tipo de atitude tomada diante das serpentes, sendo menos hostis os indivíduos mais instruídos. Pessoas com menos escolaridade apresentaram maior tendência a considerar todas as serpentes como perigosas, e estas se mostraram também mais hostis com estes animais. O maior contato com atividades científicas e educativo-ambientais parece ter sido decisivo para a maior tolerância com as serpentes por parte do grupo "PESB". A realização de atividades de educação-ambiental com a população das comunidades de Araponga pode ampliar a conscientização quanto à importância das serpentes, instruindo aqueles que ainda as consideram indiscriminadamente nocivas.

Palavras-chave: etnozoologia, escolaridade, Serpentes, Serra do Brigadeiro, comunidades rurais. 


\section{Introdução}

A falta de conhecimento que uma sociedade apresenta sobre determinadas espécies pode impulsionar seu extermínio indiscriminado (Pough et al. 2001, Barbosa et al. 2007, Baptista et al. 2008). A etnozoologia, que estuda o conhecimento tradicional do homem sobre os animais, possui enfoque nos processos de interação de cada sociedade com sua fauna local (Posey 1986, Begossi et al. 2002). Neste contexto, a etno-herpetologia investiga a interação humana com a fauna de anfíbios e répteis (Barbosa et al. 2007).

As serpentes formam um dos grupos de répteis mais diversos atualmente no mundo, com mais de 3.100 espécies viventes conhecidas (Uetz 2008). No Brasil, foram registradas mais de 370 espécies (Bérnils 2010), das quais apenas 15\% são de importância médica (famílias Elapidae e Viperidae). Apesar disso, para a maioria das pessoas as serpentes são conhecidas mais pela periculosidade de tais espécies do que pelas interações tróficas que realizam com os demais animais (Lima-Verde 1994). Como resultado, observa-se em diversas regiões do Brasil a aceitação de um estereótipo negativo para todas as serpentes, que são geralmente consideradas como "animais perigosos". Tal situação, associada a alguns aspectos da cultura popular, pode potencializar conflitos entre a espécie humana e as serpentes (Cardoso et al. 2003, Argôlo 2004), influenciando negativamente no modo como as pessoas interagem com este grupo (Vizotto 2003, Silva 2006).

As serpentes também se destacam dentro da herpetofauna como animais de grande importância etnozoológica, em razão da curiosidade, medo e fascínio que podem gerar nas pessoas (Gilmore 1986). Em nosso país, em especial nas regiões norte e nordeste, diversas espécies são utilizadas, por exemplo, na medicina tradicional e em rituais religiosos (Alves \& Pereira-Filho 2007, Alves et al. 2007, 2009, Moura \& Marques 2008). A investigação do conhecimento zoológico tradicional e das práticas zooterápicas permite uma melhor compreensão das interações do homem com o ambiente, sendo fundamental na formulação de estratégias para a conservação de recursos naturais junto a comunidades locais (Alves \& Rosa 2005, Barboza et al. 2007). Espera-se que ocorra um menor extermínio de serpentes quanto maior for a conscientização a respeito das mesmas. Dessa forma, investigar o conhecimento que uma determinada comunidade demonstra sobre a fauna local é fundamental para definir e orientar campanhas de educação ambiental que visem a subsequente conservação das espécies (Costa-Neto 2000, Santos-Fita $\&$ Costa-Neto 2007).

O presente trabalho foi realizado no Leste de Minas Gerais, região Sudeste do Brasil, com o objetivo de revelar o conhecimento popular de comunidades rurais sobre as serpentes, além das práticas adotadas em casos de acidentes ofídicos. Pretendeu-se também relacionar o nível de escolaridade dos entrevistados com a periculosidade e extermínio associado pelos mesmos às serpentes.

\section{Material e Métodos}

\section{1. Área de estudo}

O estudo foi conduzido em Araponga, município com cerca de 8.300 ha, dos quais aproxidamente $67 \%$ residem na zona rural. É localizado na mesorregião da Zona da Mata, Estado de Minas Gerais, Sudeste do Brasil (Instituto... 2009). Destaca-se na região o Parque Estadual da Serra do Brigadeiro (PESB) (20 $43^{\prime}$ S, 42 $\left.{ }^{\circ} 29^{\prime} \mathrm{W}\right)$, uma unidade de conservação de Mata Atlântica com aproximadamente 15 mil ha, situada em uma Área de Importância Biológica Especial para a conservação da herpetofauna (Drummond et al. 2005). Os moradores das comunidades rurais de Estouro, Praia D'Anta e São Joaquim, localizadas no entorno do PESB, juntamente com os funcionários da unidade de conservação, foram o foco das entrevistas.

\section{Levantamento de dados}

O levantamento de dados foi realizado entre os meses de agosto e novembro de 2008 através de entrevistas individuais semiestruturadas e livres (Chizzoti 2000), método que permite a captura de grande quantidade de informações, revelando tanto aspectos esperados como outros não previstos (Huntington 1998). As entrevistas consistiram no preenchimento de um formulário composto por questões fechadas (objetivas) e abertas (subjetivas), com o intuito de avaliar os conhecimentos básicos sobre as serpentes e os procedimentos adotados em casos de acidentes ofídicos.

Foram pré-definidos dois grupos para as entrevistas. O primeiro, denominado "Araponga", correspondeu aos moradores das comunidades de Estouro, Praia D' Anta e São Joaquim, na zona rural do município de Araponga. Essas comunidades foram escolhidas para o estudo por apresentarem um perfil de sociedades tradicionais, com base na cooperação social (Diegues et al. 2000). O segundo grupo foi composto pelos funcionários do Parque Estadual da Serra do Brigadeiro, sendo assim denominado "PESB". Optou-se pela divisão dos entrevistados em dois grupos por se tratarem de pessoas que vivem sobre situações relativamente distintas, uma vez que os funcionários do parque estão em constante contato com atividades educativoambientais que poderiam influenciar o resultado. No total foram realizadas 70 entrevistas individuais e anônimas, 50 com o grupo "Araponga" e 20 com o grupo "PESB". O número de entrevistados do grupo "PESB" foi limitado pelo número de funcionários do parque na época do estudo. Para o grupo "Araponga", a abordagem procedeuse de forma arbitrária, sendo entrevistadas aquelas pessoas que se dispunham a responder o questionário. Todas as entrevistas foram realizadas em particular, evitando assim que em locais com dois ou mais entrevistados, as respostas de um influenciassem a de outro.

Levantaram-se informações sobre o perfil social dos entrevistados, como sexo, idade, escolaridade e religião, no intuito de verificar a homogeneidade dos grupos. Cada entrevistado respondeu se já havia sido vítima de acidente ofídico e, em caso afirmativo, qual a parte do corpo atingida e quais providências foram tomadas após o acidente. A entrevista também procurou evidenciar quais os critérios utilizados pelos entrevistados para identificar as serpentes potencialmente peçonhentas (Viperidae e Elapidae). Em particular para os procedimentos de prevenção e primeiros socorros considerouse o número total de atitudes citadas, o que resultou em um número superior ao de entrevistados.

Os níveis de instrução dos entrevistados foram agrupados em escala ordinal de um a cinco, na seguinte ordem crescente de escolaridade: 1 = sem estudo e ensino primário incompleto, $2=$ ensino primário completo e ensino fundamental incompleto, $3=$ ensino fundamental completo e ensino médio incompleto, 4 = ensino médio completo ou superior incompleto, e $5=$ ensino superior completo. Informações adicionais sobre a evasão escolar para o município de Araponga foram levantadas a partir de dados do Instituto de Pesquisa Econômica Aplicada (2006).

As respostas dos entrevistados em relação às questões subjetivas foram agrupadas em categorias pré-definidas, a fim de comparação. $\mathrm{O}$ questionamento a respeito de qual atitude a ser tomada em caso de encontro eventual com uma serpente resultou em seis diferentes categorias de respostas, definidas numa escala ordinal, em ordem crescente de hostilidade para com o animal: $1=$ deixa a serpente ir embora, 2 = tenta espantá-la para o mato, ou chama a Polícia Ambiental, IBAMA (Instituto Brasileiro do Meio Ambiente e dos Recursos Naturais Renováveis), ou IEF (Instituto Estadual de Florestas), 3 = tenta capturá-la se tiver condição, $4=$ mata a serpente caso não consiga removê-la, 5 = chama alguém para matar a serpente, $6=$ mata a serpente. Optou-se pela diferenciação da categoria quatro em relação às categorias cinco e seis, dado que os entrevistados não optavam pela morte imediata da serpente na primeira opção. Desta forma, ao final da ordenação obteve-se uma escala baseada 
na hostilidade do entrevistado, com valores mínimo $=1$ (hostilidade compatível à categoria 1 ), e máximo $=5$ (hostilidade compatível às categorias 5 e 6 ). A periculosidade conferida às serpentes foi verificada através da resposta de afirmação ou negação ao fato do entrevistado considerar todas as serpentes como perigosas. Posteriormente, esses relatos foram adaptados para um modelo binomial, considerando a ausência/presença da aferição de perigo a todas as serpentes.

\section{Análise de dados}

As análises foram realizadas por meio de modelos lineares generalizados (GLM) (Crawley 2007), com posterior análise dos resíduos, de forma a conferir a adequação do modelo e distribuição. Todos os cálculos estatísticos foram efetuados no programa $\mathrm{R}$ (R Development Core Team 2008).

Foi realizada uma simplificação dos modelos através da retirada de termos não-significativos $(\mathrm{P}>0,05)$ do modelo inicial, tomados a partir das variáveis de menor deviância. Cada retirada de variável de dado modelo foi seguida por uma Análise de Deviância (ANOVA) com teste $\mathrm{F}$ (com exceção para análises com distribuição binomial, para as quais se utilizou teste $\chi^{2}$ ), de modo a recalcular a Deviância explicada pelos termos restantes. A fim de evitar interpretações decorrentes de termos redundantes, modelos distintos foram construídos e testados, cada qual compreendendo todas as variáveis explicativas, mas diferindo um dos outros pela ordem na qual as variáveis eram incluídas no modelo.

Dada a complexidade das interações sociais e culturais com fauna local optou-se, por fim, pelo uso de um modelo de diagnóstico sistêmico para sumarização das inter-relações abrangidas pelo conhecimento tradicional (Anderson \& Johnson 1997). Desta forma, no intuito de auxiliar a interpretação final, os principais resultados foram representados através de um diagrama de influência dos fatores sócio-culturais envolvidos na percepção das comunidades investigadas sobre as serpentes.

\section{Resultados}

\section{Perfil sócio-cultural}

A distribuição dos entrevistados entre os grupos "Araponga" e "PESB" não diferiu quando analisadas em relação à religião $\left(F_{1,68}=0,562 ; P=0,455\right)$, sexo $\left(F_{1,68}=2,090 ; P=0,152\right)$ e idade $\left(F_{1,68}=2,817 ; P=0,097\right)$, verificando-se um perfil homogêneo dos grupos para tais características. A maioria dos entrevistados, 74,28\% $(\mathrm{n}=52)$ correspondia a pessoas do sexo masculino, e $25,71 \%$ pessoas do sexo feminino $(n=18)$. Com relação às categorias de idade, observouse predominância de indivíduos de "25-34 anos" no grupo "PESB". A categoria “35-44 anos" correspondeu à de maior número entre o total de entrevistados $(22,85 \% ; n=16)$ (Figura 1a). Do ponto de vista religioso, constatou-se predominância de católicos $(75 \% ; \mathrm{n}=53)$, seguida por evangélicos $(17 \% ; n=12)$ e sem religião $(7 \% ; n=5)$ (Figura $1 b)$.

Verificaram-se diferenças nos níveis de escolaridade (representados pelas categorias de ensino) quando analisados em relação à idade do indivíduo $\left(F_{1,68}=17,217 ; P<0,001\right)$ e ao grupo pertencente $\left(F_{1,68}=4,839 ; P=0,031\right)$. Observam-se menores níveis de escolaridade para os indivíduos mais velhos em ambos os grupos entrevistados, e maiores níveis de escolaridade para o grupo "PESB" (Figura 2). A distribuição através das demais categorias de ensino é apresentada na Figura 3. Tais resultados corroboram a separação dos entrevistados nos grupos pré-definidos a fim de permitir comparações baseadas no nível de instrução escolar.

\section{Acidentes ofídicos, prevenção e procedimentos}

Do total de entrevistados, $11(15,71 \%)$ disseram ter sofrido algum acidente ofídico, sendo que dois destes por mais de uma vez. Apenas os membros (superiores e inferiores) foram relatados como região anatômica atingida. O pé destacou-se com 11 dos 13 casos de acidente $(84,61 \%)$, ao passo que perna e mão tiveram apenas um relato cada. Em seis dos 13 acidentes reportados $(46,15 \%)$ o indivíduo procurou auxílio médico e fez uso de soroterapia para o tratamento. Nos demais casos $(\mathrm{n}=7 ; 53,84 \%)$ o indivíduo não procurou auxílio médico ou não respondeu qual procedimento adotou.

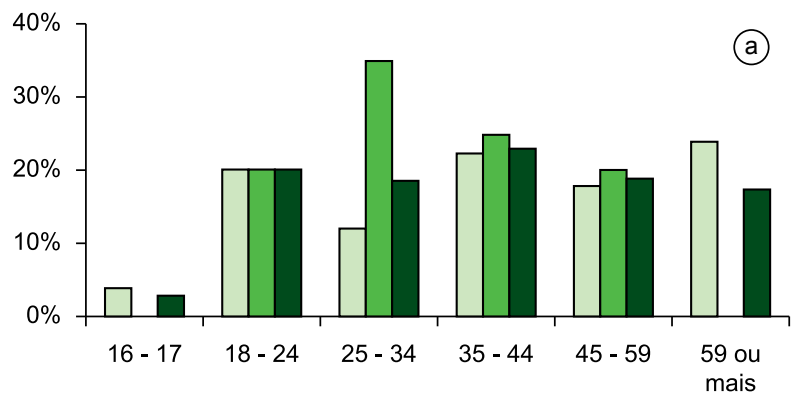

Faixa etária (em anos)

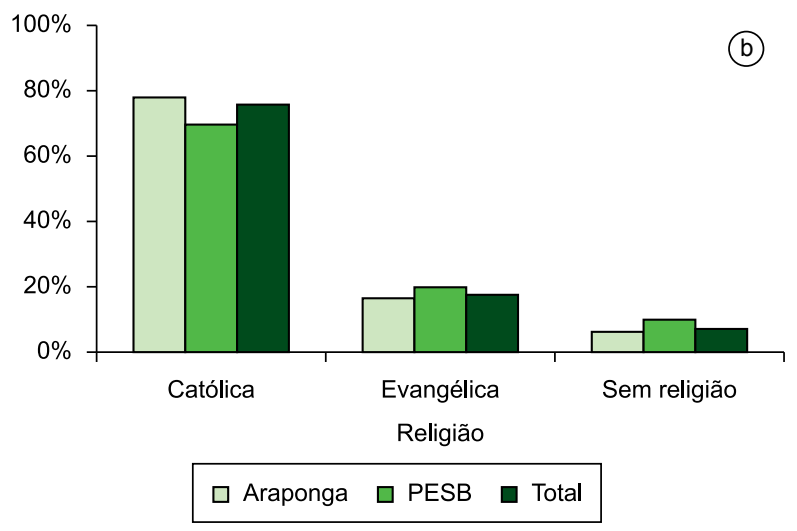

Figura 1. Distribuição percentual dos grupos de entrevistados e do total em relação à a) faixa etária; e b) religião.

Figure 1. Percentage distribution of the respondent groups and the total in relation to a) age; and b) religion.

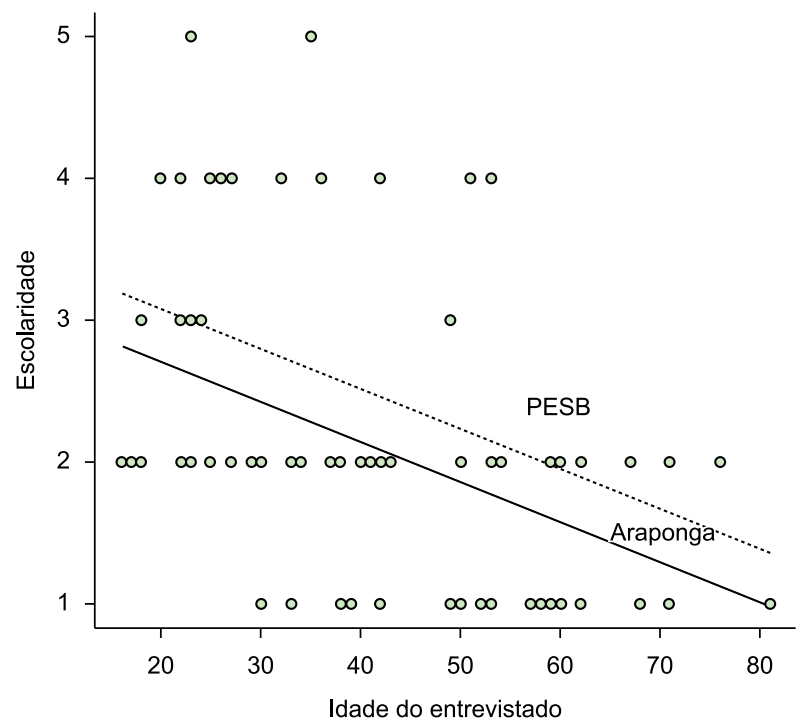

Figura 2. Escolaridade (categorias 1 a 5 segundo Materiais e Métodos) dos entrevistados em função da idade.

Figure 2. Educational level (categories 1 to 5 following Materials and Methods) of respondents in function of age. 
Dentre o total de citações de procedimentos para prevenção de acidentes com serpentes, a maioria informada $(87,15 \% ; n=95)$ é tida como eficiente (Feitosa et al. 1997) (Tabela 1). Entre os procedimentos recomendados, as medidas mais indicadas foram (alguns entrevistados indicaram mais de uma): uso de proteção para membros inferiores (bota, perneira ou calçado fechado) $(40 \% ; \mathrm{n}=38$ ); cuidado por onde anda, senta ou deita quando em ambientes propícios, (26,32\%; $\mathrm{n}=25)$; uso de equipamentos de proteção individual $(10,53 \% ; \mathrm{n}=10)$. Entre as atitudes preventivas ineficientes estão procedimentos como: orações religiosas $(28,57 \% ; n=4)$, ingestão de sementes vegetais $(14,28 \% ; n=2)$ e uso de alho nos bolsos $(7,14 \% ; n=1)$.

O número de citações de procedimentos eficientes para prevenção de acidentes ofídicos mostrou-se maior entre os entrevistados do grupo "PESB" $\left(F_{1,68}=13,251 ; P<0,001\right)$ (Figura 4a). A simplificação posterior do modelo não demonstrou relação do número de procedimentos eficientes com as categorias de ensino $\left(F_{1,67}=1,719\right.$; $P=0,194)$.

Em relação às medidas a serem tomadas em caso de ofidismo, a maioria das citações informadas pelos entrevistados $(65 \% ; n=78)$ é considerada adequada para procedimentos em caso de acidente ofídico (Cardoso et al. 2003) (Tabela 2). Entre os procedimentos indicados em caso de acidente destacaram-se os seguintes (alguns entrevistados indicaram mais de uma medida): procura por atendimento médico $(78,20 \% ; n=61)$, limpeza do local da picada com água $(11,53 \%$; $\mathrm{n}=9$ ) e identificação ou captura segura do animal causador do acidente $(7,69 \%, n=6)$. Entre os principais procedimentos contraindicados que os entrevistados relataram utilizar em caso de acidente estão o uso de torniquete no local da picada $(11,90 \% ; n=5)$, ingestão de bebida alcoólica $(9,52 \% ; n=4)$, sucção do veneno $(4,76 \% ; n=2)$ e cortar a pele na região da picada $(4,76 \% ; n=2)$.

O número de citações de procedimentos eficientes em caso de acidente ofídico mostrou-se maior no grupo "PESB" $\left(F_{1,68}=5,171\right.$; $P=0,026$ ) (Figura 4b) e nos indivíduos das categorias superiores de ensino $\left(F_{1,67}=4,569 ; P=0,036\right)$.

Tabela 1. Número e percentagem de citações de procedimentos cientificamente considerados eficientes e ineficientes, relatados para prevenção de acidentes ofídicos pelos grupos "Araponga" e "PESB".

Table 1. Number and percentage of citations of procedures for prevention of snakebites considered considered to be efficient and inefficient by science, reported by "Araponga" and "PESB" groups.

\begin{tabular}{|c|c|c|c|c|c|c|}
\hline \multirow{2}{*}{$\begin{array}{c}\text { Tipo de procedimento } \\
\text { utilizado para } \\
\text { prevenir acidente } \\
\end{array}$} & \multicolumn{2}{|c|}{ Araponga } & \multicolumn{2}{|c|}{ PESB } & \multicolumn{2}{|c|}{ Total } \\
\hline & $\mathbf{N}$ & $\%$ & $\mathbf{N}^{0}$ & $\%$ & $\mathbf{N}$ & $\%$ \\
\hline Atitudes eficientes & 58 & 80,55 & 37 & 100,00 & 95 & 87,15 \\
\hline Atitudes ineficientes & 14 & 19,45 & 0 & 0,00 & 14 & 12,85 \\
\hline Total & 72 & 100,00 & 37 & 100,00 & 109 & 100,00 \\
\hline
\end{tabular}

Tabela 2. Número e percentagem de citações de procedimentos cientificamente considerados eficientes e ineficientes, relatados pelos entrevistados dos grupos "Araponga" e "PESB" em caso de acidente ofídico.

Table 2. Number and percentage of citations of procedures considered to be efficient and inefficient by science, reported by "Araponga" and "PESB" groups in case of snakebite.

\begin{tabular}{|c|c|c|c|c|c|c|}
\hline \multirow{2}{*}{$\begin{array}{l}\text { Procedimento em } \\
\text { caso de acidente }\end{array}$} & \multicolumn{2}{|c|}{ Araponga } & \multicolumn{2}{|c|}{ PESB } & \multicolumn{2}{|c|}{ Total } \\
\hline & $\mathbf{N}$ & $\%$ & $\mathbf{N}^{\mathbf{o}}$ & $\%$ & $\mathbf{N}$ & $\%$ \\
\hline Atitudes eficientes & 51 & 56,67 & 27 & 90,00 & 78 & 65,00 \\
\hline Atitudes ineficientes & 39 & 43,33 & 3 & 10,00 & 42 & 35,00 \\
\hline Total & 90 & 100,00 & 30 & 100,00 & 120 & 100,00 \\
\hline
\end{tabular}

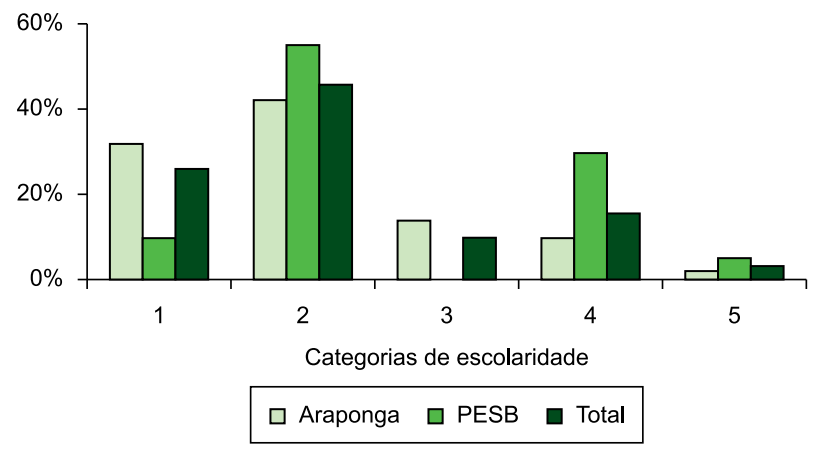

Figura 3. Distribuição percentual dos grupos de entrevistados e do total em relação às categorias de escolaridade: 1) sem estudo ou ensino primário incompleto; 2) ensino primário completo ou fundamental incompleto; 3 ) ensino fundamental completo ou médio incompleto; 4) ensino médio completo ou superior incompleto; e 5) ensino superior completo.

Figure 3. Percentage distribution of groups of respondents and the total for the following categories of education: 1) no education or incomplete primary education; 2) complete primary education or incomplete elementary school; 3) complete elementary school or incomplete high school; 4) complete high school and incomplete higher education; and 5) college degree.
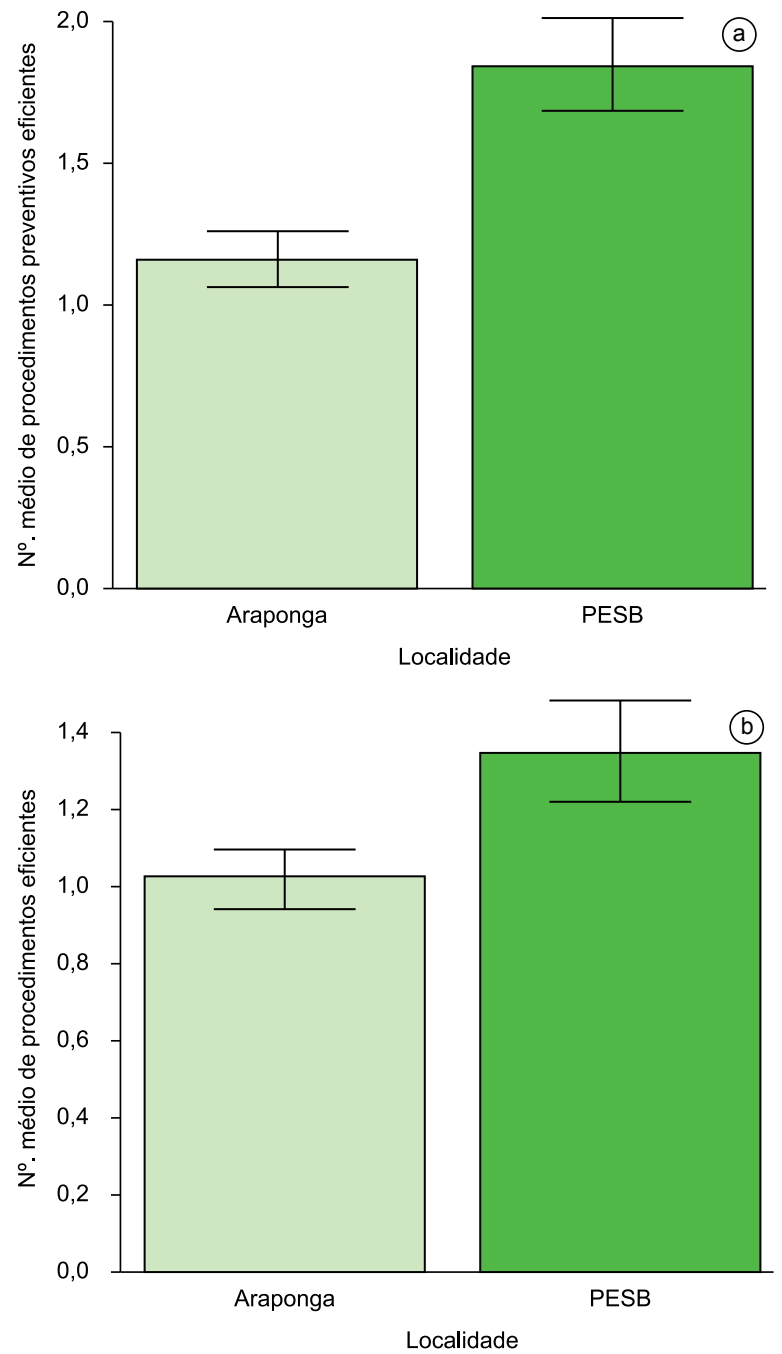

Figura 4. a) Número médio de procedimentos eficientes para prevenção de acidentes ofídicos; e b) número médio de procedimentos eficientes em caso acidentes ofídicos, relatados pelos grupos "Araponga" e "PESB".

Figure 4. a) Average number of efficient procedures for prevention of snakebites; and b) average number of efficient procedures in case snakebites, reported by the groups "Araponga" and "PESB". 
O uso de práticas da medicina religiosa (procura de curandeiros ou benzedeiros) mostrou-se pouco frequente em ambas as classes de entrevistados, sendo adotado por 21,42\% $(\mathrm{n}=15)$ do total ("Araponga", $\mathrm{n}=12 ; 24,0 \%$ e "PESB", $\mathrm{n}=3 ; 15,0 \%)$. A utilização de produtos vegetais mostrou-se o procedimento mais comum entre as práticas de medicina popular (e.g. uso de macerados de alho e seiva de mamão no local da picada; ingestão de infusões com gramíneas e ervas).

\section{Etno-herpetologia}

Metade dos entrevistados $(50 \% ; n=35)$ respondeu não saber diferenciar uma serpente peçonhenta de uma não-peçonhenta ("Araponga", $\mathrm{n}=27 ; 54,0 \%$ e "PESB", $\mathrm{n}=8 ; 40,0 \%$ ). Os demais relataram os caracteres a seguir como determinantes na identificação de uma serpente como peçonhenta. Grupo "Araponga": cabeça triangular $(\mathrm{n}=7 ; 21,87 \%)$, comportamento de enrodilhar $(\mathrm{n}=6$; $18,75 \%)$, cabeça achatada $(n=4 ; 12,5 \%)$, padrão de coloração $(n=3$; $9,04 \%)$, cauda que "afina bruscamente" $(n=2 ; 6,25 \%)$, pupila vertical $(\mathrm{n}=2 ; 6,25 \%)$ e presença de chocalho $(\mathrm{n}=2 ; 6,25 \%)$. Para o grupo "PESB": cabeça triangular $(\mathrm{n}=6 ; 30 \%)$, presença de fosseta loreal $(n=4 ; 20 \%)$, padrão de coloração $(n=3 ; 15 \%)$ e comportamento de enrodilhar $(n=2 ; 10 \%)$. Entre algumas características citadas apenas uma vez em cada grupo estão: escamas ásperas, cabeça destacada da região do corpo e presença de dente inoculador de veneno.

A maioria dos entrevistados $(65,71 \% ; n=46)$ indicou a estação chuvosa e/ou quente como determinante na atividade das serpentes. Outros $11,14 \%(\mathrm{n}=8)$ associaram a estação fria à maior facilidade de encontro, enquanto que $7,43 \%(n=5)$ afirmaram que a época de colheita do café, cultura predominante na região, estaria relacionada à maior probabilidade de encontro com uma serpente. Os demais entrevistados $(12,85 \% ; \mathrm{n}=9)$ não opinaram sobre a época com maior taxa de encontro de ofídios.

O tipo de decisão tomada em caso de encontro com alguma serpente mostrou-se mais hostil entre o grupo "Araponga", no qual $46 \%(n=23)$ dos entrevistados afirmaram que a atitude tomada seria "matar a serpente". Por outro lado, no grupo "PESB", 65\% (n=13) dos relatos consistiam em "deixar a serpente ir embora" ou "espantá-la para o mato" (Figura 5). O nível de hostilidade (associado ao tipo de atitude tomada diante de uma serpente) foi maior tanto para os relatos do grupo "Araponga" $\left(F_{1,68}=9,960 ; P=0,002\right)$ (Figura 6a), quanto entre os indivíduos que consideraram todas as serpentes perigosas $\left(F_{1,67}=4,274\right.$; $P=0,042$ ) (Figura 6b). Embora o grupo "Araponga" tenha apresentado um menor nível de instrução, a hostilidade apresentada não se mostrou relacionada com a categoria de ensino $\left(F_{1,68}=0,680 ; P=0,412\right)$ ou com a idade do indivíduo $\left(F_{1,68}=3,206 ; P=0,077\right)$.

Com relação à periculosidade associada aos ofídios, $48 \%(\mathrm{n}=24)$ dos entrevistados do grupo "Araponga" afirmou acreditar que todas as serpentes são perigosas, sendo a mesma resposta observada para $30 \%(\mathrm{n}=6)$ dos indivíduos do grupo "PESB". Apesar disso, a mesma não se diferenciou entre os grupos $\left(\chi_{68}^{2}=91,728 ; P=0,072\right)$. Nota-se uma redução do número de indivíduos que consideram as serpentes perigosas (periculosidade) entre as categorias de ensino mais elevadas $\left(\chi_{68}^{2}=77,866 ; P<0,001\right)$ (Figura 7a). A simplificação posterior do modelo não indicou a idade do indivíduo como explicativa de variação adicional $\left(\chi_{67}^{2}=77,111 ; P<0,385\right)$, embora os indivíduos mais novos tenham raramente definido as serpentes como perigosas (Figura $7 b$ ).

\section{Discussão}

\section{Perfil sócio-cultural}

Os dados referentes ao perfil cultural e social das comunidades investigadas revelam uma caracterização religiosa entre os entrevistados, fato este que contribui para a classificação dos moradores das comunidades de Araponga como sociedades tradicionais (Diegues et al. 2000). A não distinção dos grupos entre as categorias de religião, sexo e idade permite inferir que estes são similares nesse quesito (Figura 2), e que, embora apresentem diferenças em relação aos níveis de escolaridade, ainda podem ser considerados relativamente homogêneos.
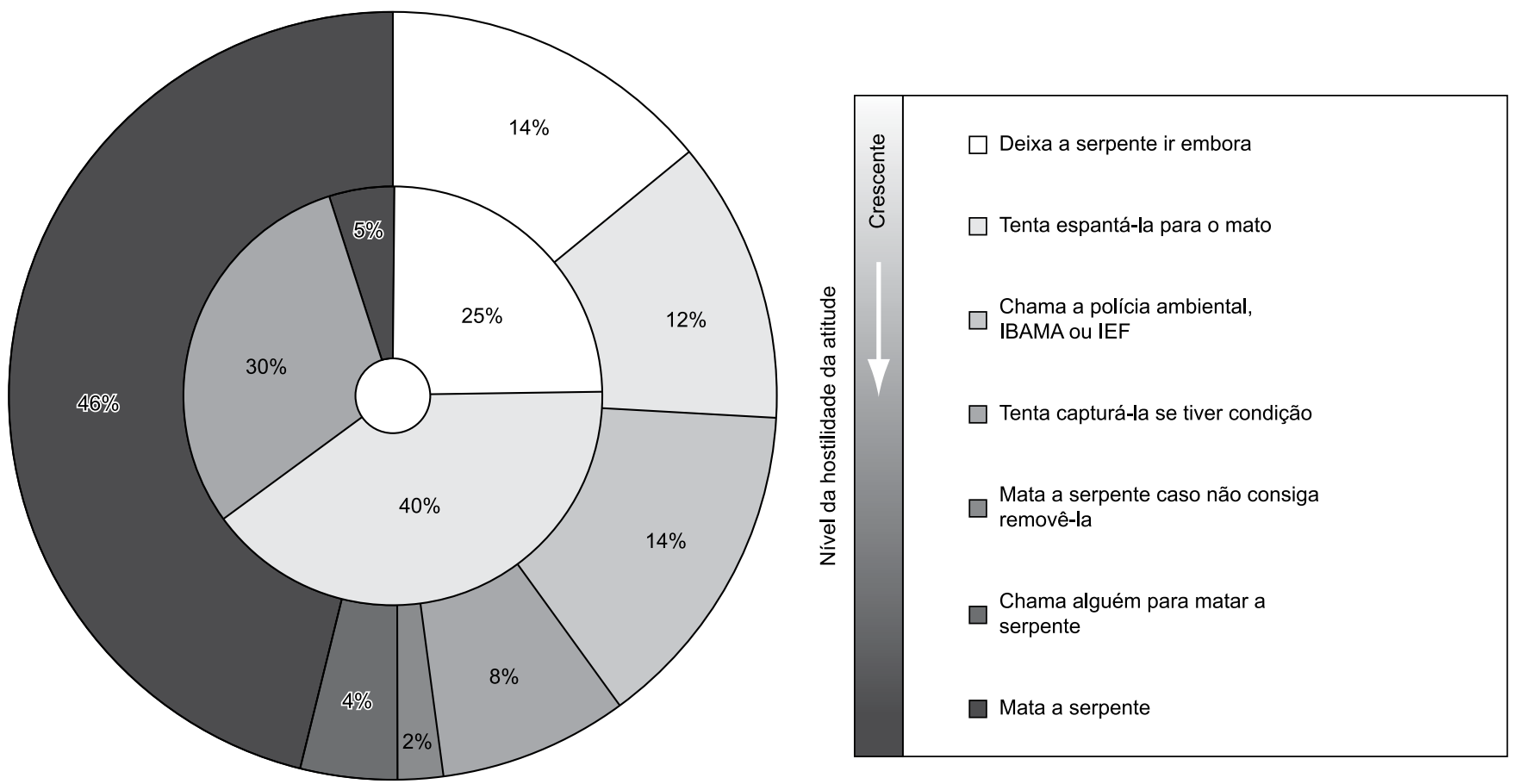

Figura 5. Distribuição percentual da atitude dos entrevistados ao se depararem com uma serpente. Gráfico externo: grupo "Araponga". Gráfico interno: grupo "PESB".

Figure 5. Percentage distribution of respondents attitude when faced with a snake. External graph: group "Araponga". Internal graph: group "PESB". 
Moura, M.R. et al.
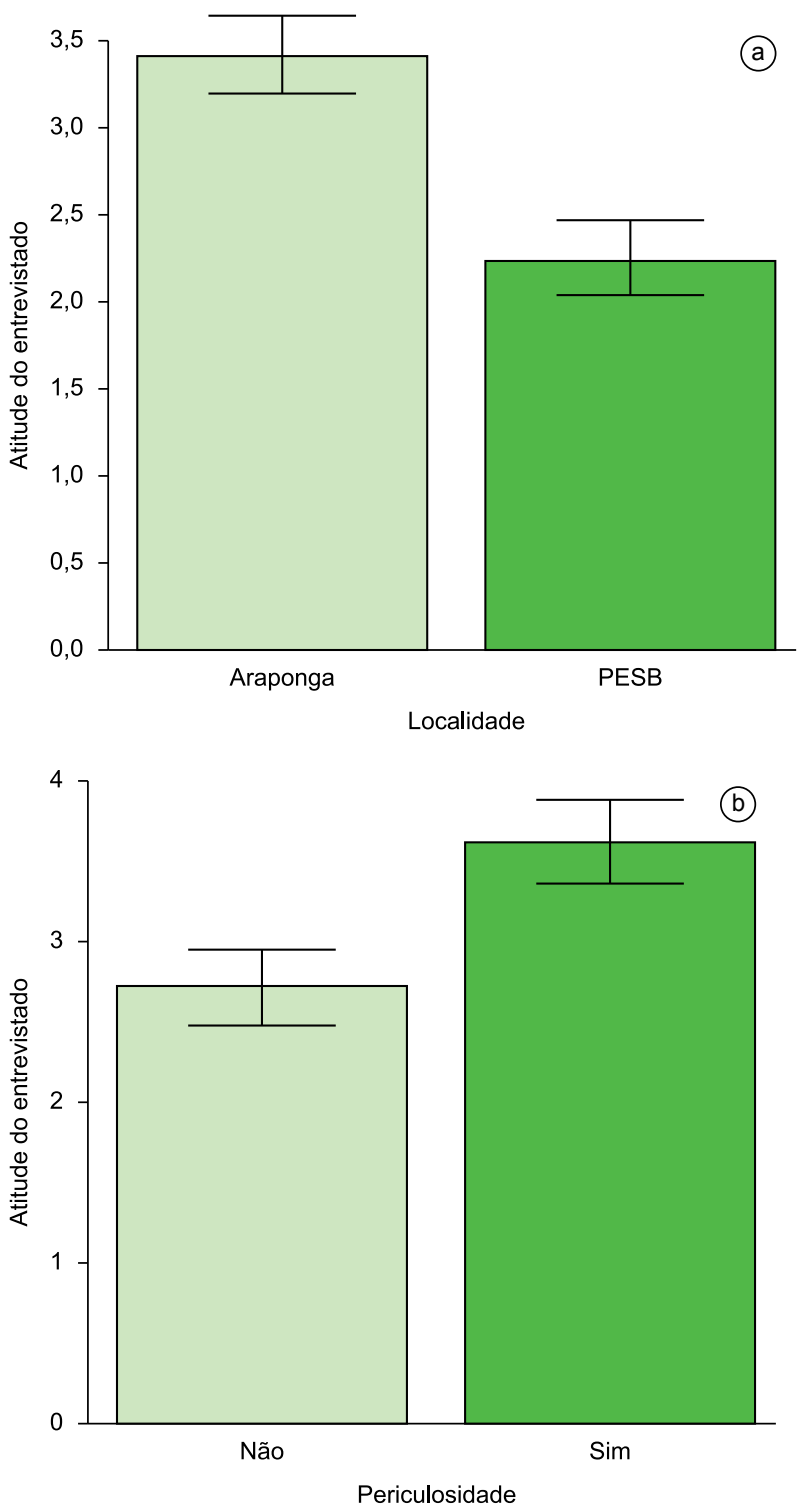

Figura 6. a) Nível de hostilidade da atitude adotada pelos entrevistados dos grupos "Araponga" e "PESB" quando em eventual encontro com uma serpente (com base nas categorias definidas em Material e Métodos); e b) nível de hostilidade da atitude dos entrevistados em relação à sua opinião sobre todas as serpentes serem perigosas (Periculosidadep.

Figure 6. a) Level of hostility of the attitude adopted by the respondents of the groups "Araponga" and "PESB" when faced with a snake (based on categories defined in Material and Methods); and b) level of hostility of the attitudes of respondents regarding their views that all snakes are dangerous (dangerousness).

Os dados referentes à escolaridade demonstraram uma diminuição do nível de instrução do entrevistado quando analisado em relação à sua idade (Figura 2). O menor nível de instrução entre os entrevistados de maior idade pode estar relacionado à alta evasão escolar observada no passado. Segundo dados do Instituto de Pesquisa Econômica Aplicada (IPEA), a evasão escolar para o município de Araponga em 1991 era de $38,04 \%$ para pessoas de 7 a 14 anos, e de $85,58 \%$ entre 15 e 17 anos. No ano 2000, essa evasão atingia 10,42\% dos indivíduos de 7 a 14 anos e $54,89 \%$ de 15 a 17 anos. Em 1991, 76,4\% da população acima de 25 anos não haviam completado a quarta série do ensino primário, contra 66,2\% em 2000 (Instituto... 2006). A redução da
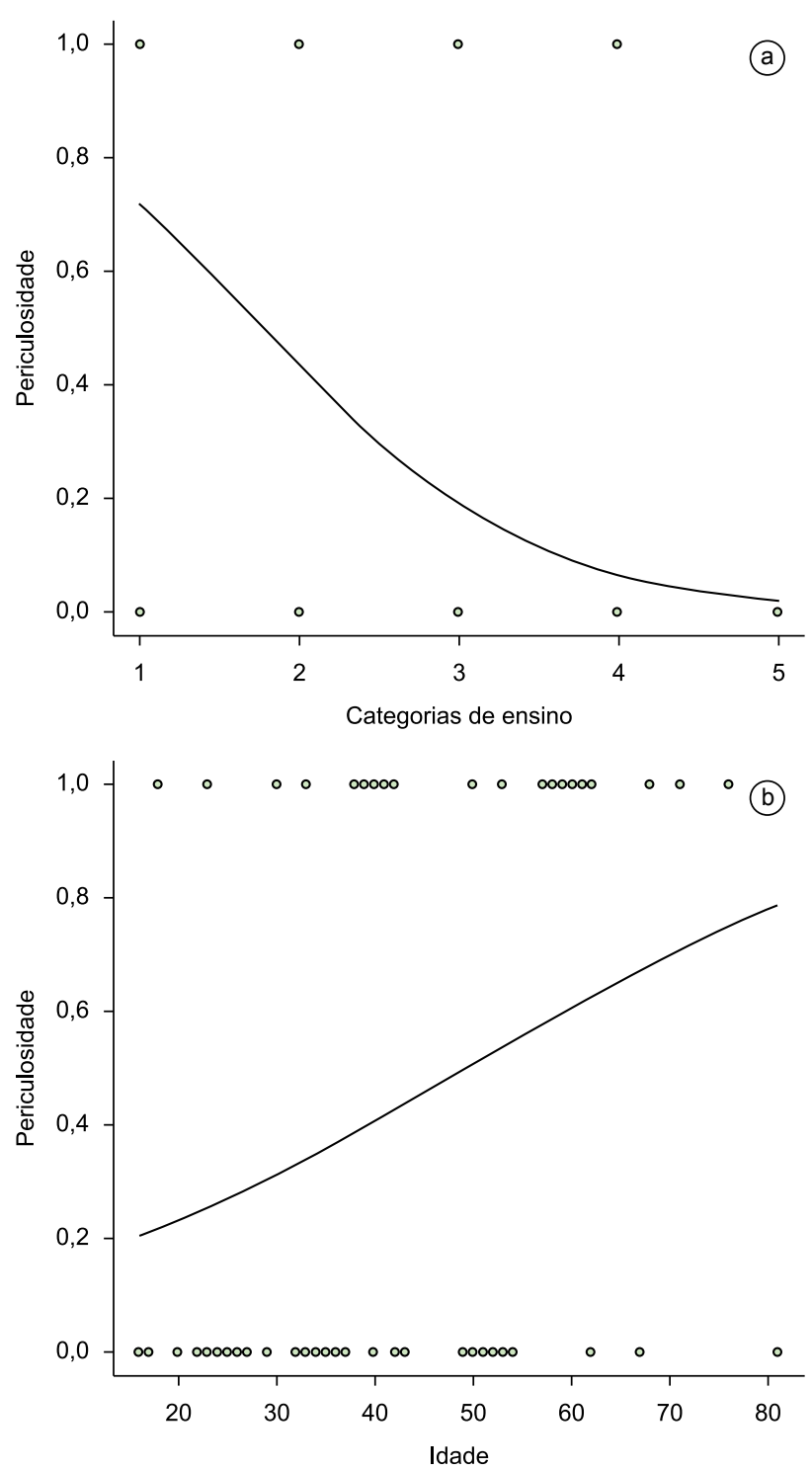

Figura 7. Periculosidade (ausência/presença de indicação de todos os ofídios como perigosos) associada às serpentes em relação à a) categoria de ensino; e b) idade do entrevistado.

Figure 7. Dangerousness (absence/presence of indication that all snakes are dangerous) associated to snakes in relation to the a) educational category; and b) age of respondent.

evasão escolar em épocas recentes sugere um reflexo de melhorias nas condições sociais na região, o qual teria proporcionado maior acesso dos jovens às escolas. Tais melhorias no ensino mostraram-se importantes no nível de conscientização dos entrevistados, uma vez que aqueles mais instruídos apresentaram um melhor conhecimento tanto sobre atitudes corretas em acidentes ofídicos quanto em relação à biologia das serpentes.

\section{Acidentes ofídicos, prevenção e procedimentos}

A predominância dos membros inferiores como a região anatômica mais atingida nos casos de acidentes ofídicos é observada em diversos estudos relacionados ao ofidismo (e.g. Feitosa et al. 1997, Ribeiro et al. 1998, Pinho et al. 2004). Esse padrão pode ser explicado pelo hábitat terrícola das principais espécies de importância médica associado à baixa frequência no uso de calçados fechados durante 
as atividades agrícolas. A adequação de vestuário (botas, perneiras, luvas e outros) durante o trabalho no campo poderia prevenir grande parte dos acidentes (Feitosa et al. 1997, Marques et al. 2004).

A simplificação do modelo utilizado nas análises não demonstrou relação entre o número de procedimentos preventivos recomendáveis e as categorias de ensino, demonstrando que o grau de escolaridade aparentemente não influenciou no conhecimento de medidas preventivas ao ofidismo. Com relação aos primeiros socorros, a maioria dos entrevistados do grupo "PESB" também demonstrou possuir conhecimento adequado sobre os procedimentos eficientes a serem tomados em caso de acidente ofídico $(90 \%$; $\mathrm{n}=27$ dos procedimentos citados), contrastando com o grupo "Araponga", no qual se observou menor número de atitudes eficientes informadas $(56,67 \% ; \mathrm{n}=51)$ (Tabela 2). Possivelmente, o maior grau de escolaridade influencia negativamente na crença de tratamentos por meios de práticas tradicionais (medicina tradicional e curandeirismo).

A adoção de práticas ineficientes em caso de acidente ofídico pode resultar no agravamento do quadro clínico do acidentado. Entre as atitudes inadequadas informadas pelos entrevistados está o uso de torniquete (garrote), o qual é ineficiente para impedir a propagação do veneno pelo sistema circulatório do acidentado (Grenard 2000). Em caso de acidente botrópico, por exemplo, a utilização deste método pode resultar em necrose e/ou gangrena no local da picada, com posterior evolução de complicações que podem levar à amputação do membro garroteado ou mesmo no óbito do indivíduo (Pinho et al. 2004).

A ausência do uso de práticas zooterápicas para o tratamento de acidentes ofídicos nas comunidades estudadas pode estar relacionada a processos políticos, econômicos e históricos que teriam desestimulado a disseminação dessas práticas (Davis \& Wagner 2003). De acordo com os relatos obtidos, a procura de ajuda de curandeiros ou benzedeiros para o tratamento de acidentes ofídicos consistia prática comum no passado. A diminuição desse tipo de prática pode ser um reflexo de melhorias recentes no sistema de saúde e/ou dos meios de transporte, que facilitariam a busca por auxílio médico.

\section{Relação entre seres humanos e serpentes}

A identificação errônea de serpentes potencialmente peçonhentas (Viperidae e Elapidae) pode contribuir para o aumento do número de acidentes. A citação de características como cabeça com formato triangular, cabeça achatada e pupila vertical mostrou-se comum entre as pessoas que disseram saber identificar uma serpente peçonhenta. Apesar disso, alguns métodos informados podem auxiliar na prevenção de acidentes, como o comportamento de enrodilhar (típico da maioria das espécies peçonhentas) e a presença de fosseta loreal, característica marcante dos viperídeos americanos (Fundação..., 2001, Campbell \& Lamar 2004)

O conhecimento dos entrevistados sobre a atividade sazonal das serpentes é condizente com o observado na literatura específica para ofídios da Mata Atlântica e Pantanal (e.g. Marques et al. 2004, 2005). A afirmação de que a época da colheita do café (abril a junho) seria mais propícia à observação de serpentes pode estar relacionada ao fato de que, durante a colheita, o indivíduo estaria em atividades no campo por um período maior de tempo, o que aumentaria suas chances de encontro com esses animais.

As atitudes humanas direcionadas aos animais são influenciadas pela percepção, identificação e classificação que cada sociedade faz da fauna local (Drews 2002). Quando observados individualmente, os grupos "Araponga" e "PESB" diferiram no tipo de atitude tomada em caso de um eventual encontro com uma serpente, provavelmente em razão dos diferentes contextos educativo-ambientais aos quais cada grupo está sujeito (Figuras 5 e 6a). Embora tenham diferido em relação às categorias de escolaridade, a hostilidade (determinada pelas categorias de atitudes em caso de encontro com uma serpente) não apresentou relação significativa com a escolaridade. Entretanto, esta mesma hostilidade mostrou-se relacionada com o conhecimento sobre a periculosidade das serpentes, sugerindo um importante papel da educação ambiental na redução da hostilidade do comportamento humano para com as serpentes (Figura 8).

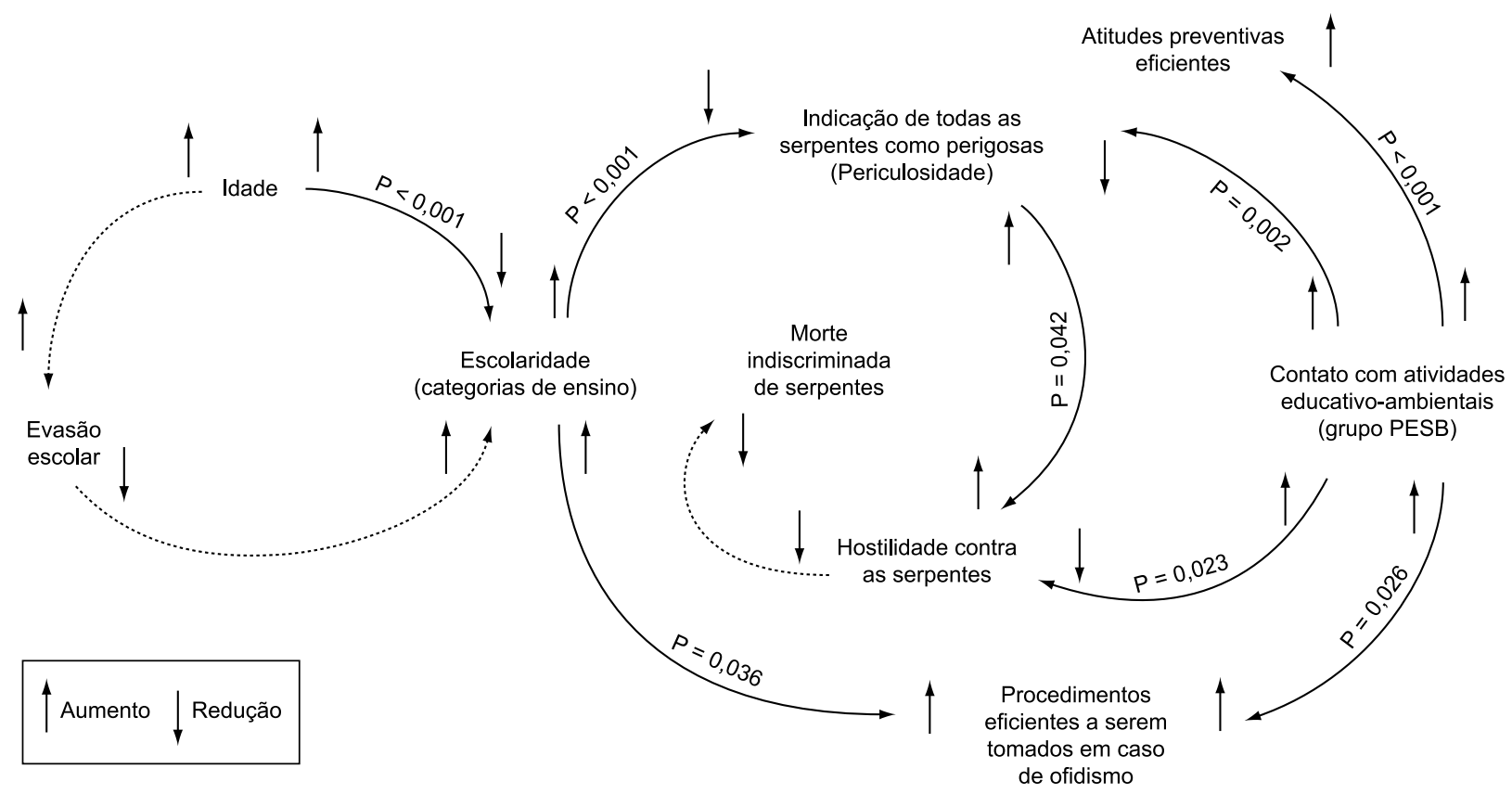

Figura 8. Diagrama de influência dos fatores sócio-culturais na percepção dos entrevistados sobre serpentes. Traços uniformes representam considerações diretamente observadas no presente estudo. Traços descontínuos representam considerações inferidas com base na literatura.

Figure 8. Diagram of the influence of socio-cultural factors in perceptions of the interviewee concerning on snakes. Uniform lines represent considerations observed in this study. Dashed lines represent considerations inferred from literature. 
A opção de matar uma serpente foi justificada pelo fato de o indivíduo a considerar perigosa. Segundo Nolan \& Robbins (2001), o nível de empatia para com os animais é determinante no comportamento humano diante dos mesmos. Tal associação é facilmente observada entre os entrevistados, uma vez que atitudes mais hostis mostraram-se diretamente relacionadas aos entrevistados que associavam perigo às serpentes (Figura 6b). De acordo com Drews (2002), quanto maior for o fascínio que um indivíduo possui sobre determinado animal, maior será seu conhecimento sobre a biologia do mesmo. Se a intenção de matar uma serpente for interpretada como um sentimento antagônico à atração pelo animal, pode-se verificar um menor nível de instrução (geralmente observado entre os indivíduos de maior idade) entre os entrevistados que apresentaram repulsa pelas serpentes (Figura 7). Sabe-se que na região do presente estudo ocorrem ao menos duas espécies de serpentes de importância médica (Bothrops jararaca e Micrurus frontalis). No Brasil, a presença de serpentes no mesmo ambiente gera conflitos com a espécie humana (Argôlo 2004), levando à generalização de um estereótipo negativo para as serpentes, o que contribui para a morte indiscriminada de espécies, peçonhentas ou não.

A realização de programas de conscientização ambiental é fundamental para reforçar a necessidade de conservação dos recursos biológicos (Torres et al. 2009). Os maiores níveis de escolaridade verificados para o grupo "PESB" associados ao maior contato com atividades educativo-ambientais podem ter influenciado o conhecimento zoológico tradicional dos entrevistados, dado que este é situacional e modificável (Santos-Fita \& Costa-Neto 2007). Consequentemente, esperara-se melhor interação entre humanos e serpentes enquanto ocorram incentivos educativo-ambientais que alterem a percepção das comunidades rurais sobre a fauna, podendo resultar em maior qualidade de vida para estas comunidades (Davis \& Wagner 2003). É importante ressaltar que a educação ambiental exige tempo e ações em longo prazo que devem levar em conta o contexto local, o respeito às diversidades e a adoção de abordagens participativas (Ministério do Meio Ambiente 2002).

\section{Conclusão}

A utilização de dois grupos distintos no estudo permitiu a identificação de diferenças nas respostas dos entrevistados. Ambos os grupos mostraram-se similares para características relacionados a religião, sexo e idade dos entrevistados. As práticas zooterápicas estão em crescente desuso pelas comunidades rurais do entorno de Araponga. O grupo "PESB" mostrou-se menos hostil em relação às possíveis atitudes diante das serpentes, provavelmente devido aos maiores índices de escolaridade desses entrevistados, e principalmente ao seu maior contato com atividades científicas e educativoambientais. O perigo que o entrevistado associa aos ofídios também contribuiu para uma maior hostilidade com esses animais. Indivíduos das categorias de ensino mais elevadas indicaram não considerarem todas as serpentes como perigosas, assim como a maioria dos indivíduos mais novos. Na região do entorno da Serra do Brigadeiro, a repulsa da população em relação às serpentes provavelmente contribui para a morte indiscriminada desses animais. Torna-se então necessária a realização de práticas de educação ambiental com a população, para sua conscientização quanto à importância das serpentes. A realização de novos estudos etnozoológicos pode contribuir para elucidar quais fatores sociais e culturais são determinantes na relação do homem com a fauna.

\section{Agradecimentos}

À Adriana C. Rodrigues, Carolina Coelho Augusto Silva e Pâmella Albinati Oliveira pelo auxílio nas entrevistas, e Rossana
Modolin pela revisão do Inglês. A Ricardo Ribeiro de Castro Solar pela colaboração com as análises estatísticas. Ao Departamento de Biologia Animal, juntamente com a Garagem Central da Universidade Federal de Viçosa, pelo apoio logístico à realização do trabalho. Aos três revisores anônimos pela leitura crítica e sugestões feitas. Agradecemos especialmente a todas as pessoas de Araponga e do Parque Estadual da Serra do Brigadeiro que colaboraram de forma essencial para este estudo por meio das entrevistas.

\section{Referências Bibliográficas}

ALVES, R.R.N. \& PEREIRA-FILHO, G.A. 2007. Commercialization and use of snakes on North and Northeastern Brazil: implications for conservation and management. Biod. Conserv. 16:969-985.

ALVES, R.R.N. \& ROSA, I.L. 2005. Why study the use of animal products in traditional medicines? J. Ethnobiol. Ethnomed. 1:1-5.

ALVES, R.R.N., LÉO NETO, N.A., SANTANA, G.G., VIEIRA, W.L.S. \& ALMEIDA, W.O. 2009. Reptiles used for medicinal and magic religious purposes in Brazil. Appl. Herpet. 6:257-274.

ALVES, R.R.N., PEREIRA-FILHO, G.A. \& LIMA, Y.C.C. 2007. Snakes used in ethnomedicine in Northeast Brazil. Environ. Develop. Sustain. 9:455-464.

ANDERSON, V. \& JOHNSON, L. 1997. Systems thinking basics: from concepts to causal loops. Pegasus Communications, Cambridge.

ARGÔLO, A.J.S. 2004. As serpentes dos cacauais do sudeste da Bahia. Editora da UESC, Ilhéus.

BAPTISTA, G.C.S., COSTA-NETO, E.M. \& VALVERDE, M.C.C. 2008. Diálogo entre concepções prévias dos estudantes e conhecimento científico escolar: relações sobre os Amphisbaenia. Rev. Iberoam. Educ. 47:1-16.

BARBOSA, A.R., NISHIDA, A.K., COSTA, E.S. \& CAZÉ, A.L.R. 2007. Abordagem etnoherpetológica de São José da Mata - Paraíba - Brasil. Rev. Biol. Ciênc. Terra 7(2):117-123.

BARBOZA, R.R.D., SOUTO, W.M.S \& MOURÃO, J.S. 2007. The use of zootherapeutics in folk veterinary medicine in the district of Cubati. J. Ethnobiol. Ethnomed 32(3):1-14.

BEGOSSI, A., HANAZAKI, N. \& SILVANO, R.A.M. 2002. Ecologia humana, etnoecologia e conservação. In Métodos de coleta e análise de dados em etnobiologia, etnoecologia e disciplinas correlatas. In Anais do Seminário de Etnobiologia e Etnoecologia do Sudeste (M.C.M. Amorozo, L.C. Ming \& S.M.P. Silva, ed.). UNESP, Rio Claro.

BÉRNILS, R.S. 2010. Brazilian Reptiles - List of species. Sociedade Brasileira de Herpetologia http://www.sbherpetologia.org.br/checklist/ repteis.htm (último acesso em 05/05/2010).

CAMPBELL, J.A. \& LAMAR, W.W. 2004. The Venomous reptiles of the Western Hemisphere. Cornell University, Ithaca.

CARDOSO, J.L.C., FRANÇA, F.P.S., WEN, F.H, MÁLAQUE, C.M.S. \& HADDAD-Jr, V. 2003. Animais peçonhentos no Brasil. Biologia, clínica e terapêutica dos acidentes. Sarvier, São Paulo.

CHIZZOTI, A. 2000. Pesquisa em Ciências Humanas e Sociais. 4. ed. Cortez, São Paulo.

COSTA-NETO, E.M. 2000. Conhecimento e usos tradicionais de recursos faunísticos por uma comunidade afro-brasileira: resultados preliminares. Interciencia 25(9):423-431.

CRAWLEY, M.J. 2007. The R Book. John Wiley \& Sons, West Sussex.

DAVIS, A. \& WAGNER, J.R. 2003. Who knows? On the importance of identifying "Experts" when reserching local ecological knowledge. Human Ecol. 31(3):463-489.

DIEGUES, A.C., ARRUDA, R.S.V., SILVA, V.C.F., FIGOLS, F.A. \& ANDRADE, D. 2000. Os saberes tradicionais e a biodiversidade no Brasil. Ministério do Meio Ambiente, Brasília.

DREWS, C. 2002. Attitudes, knowledge and wild animals as pets in Costa Rica. Anthrozoös 15(2):119-138. 
DRUMMOND, G.M., MARTINS, C.S., MACHADO, A.B.M., SEBAIO, F.A \& ANTONINI, Y. 2005. Biodiversidade em Minas Gerais: um atlas para a conservação. Fundação Biodiversitas, Belo Horizonte.

FEITOSA, R.F.G., MELO, I.M.L.A. \& MONTEIRO, H.S.A. 1997. Epidemiologia dos acidentes por serpentes peçonhentas no Estado do Ceará - Brasil. Rev. Soc. Bras. Med. Trop. 30(4):295-301.

FUNDAÇÃO NACIONAL DE SAÚDE - FUNASA. 2001. Manual de diagnóstico e tratamento de acidentes por animais peçonhentos. Ministério da Saúde, Brasília.

GILMORE, R.M. 1986. Fauna e etnozoologia da América do Sul Tropical. In Suma etnológica Brasileira (D. Ribeiro, ed.). FINEP/Vozes, Rio de Janeiro, p.189-233.

GRENARD, S. 2000. Veno and Arterio-occlusive tourniquets are not only harmful, they are unnecessary. Toxicon 38:1305-1306.

HUNTINGTON, H.P. 1998. Observations on the utility of the Semi-directive interview for documenting traditional ecological knowledge. Artic 51(3):237-242.

INSTITUTO BRASILEIRO DE GEOGRAFIA E ESTATÍSTICA - IBGE. 2009. Cidades@: Araponga. http://www.ibge.gov.br/cidadesat/ topwindow.htm?1 (último acesso em: 19/08/2010).

INSTITUTO DE PESQUISA ECONÔMICA APLICADA - IPEA. 2006 Ipeadata: dados sociais. http://www.ipeadata.gov.br/ipeaweb.dll/ipeadata ?SessionID=393347814\&Tick=1239502182559\&VAR_FUNCAO=Ser Temas(1413839210)\&Mod=S (último acesso em 18/05/ 2010.

LIMA-VERDE, J.S. 1994. Por que não matar as nossas cobras. In Herpetologia no Brasil I (L.B. Nascimento, A.T. Bernardes \& G.A. Cotta, ed.). PUC/ Biodiversitas, Belo Horizonte, p.92-101.

MINISTÉRIO DO MEIO AMBIENTE - MMA. 2002. Biodiversidade brasileira: avaliação e identificação de áreas e ações prioritárias para a conservação, utilização sustentável e repartição dos benefícios da biodiversidade nos biomas brasileiros. MMA/SBF, Brasília.

MARQUES, O.A.V., ETEROVIC, A. \& SAZIMA, I. 2004. Snakes of the Brazilian Atlantic forest: an illustrated field guide for the Serra do Mar Range. Holos, Ribeirão Preto.

MARQUES, O.A.V., ETEROVIC, A., STRÜSSMANN, C. \& SAZIMA, I. 2005. Serpentes do Pantanal: guia ilustrado. Holos, Ribeirão Preto.
MINISTÉRIO DO MEIO AMBIENTE - MMA. 2002. Biodiversidade brasileira: avaliação e identificação de áreas e ações prioritárias para a conservação, utilização sustentável e repartição dos benefícios da biodiversidade nos biomas brasileiros. MMA/SBF, Brasília.

MOURA, F.B.P. \& MARQUES, J.G.W. 2008. Zooterapia popular na Chapada Diamantina: uma medicina incidental? Rev. C. S. Col. 13(2):2179-2188.

NOLAN, J.M. \& ROBBINS, M.C.E. 2001. Emotional meaning and the cognitive organization of ethnozoological domains. J. Ling. Anthrop. 11(2):240-249.

PINHO, F.M.O., OLIVEIRA, E.S. \& FALEIROS, F. 2004. Acidente ofídico no estado de Goiás. Rev. Ass. Med. Bras. 50:93-96.

POSEY, D.A. 1986. Etnobiologia: teoria e prática. In Suma etnológica Brasileira (D. Ribeiro, ed.). FINEP/Vozes, Rio de Janeiro, p.15-25.

POUGH, F.H., ANDREWS, R.M., CADLE, J.E., CRUMP, M.L., SAVITZKY, A.H. \& WELLS, K.D. 2001. Herpetology. Pretice Hall, New Jersey.

R DEVELOPMENT CORE TEAM. 2008. R: A language and environment for statistical computing. R Foundation for Statistical Computing, Vienna.

RIBEIRO, L.A., ALBUQUERQUE, M.J., CAMPOS, V.F P., KATZ, G., TAKAOKA, N.Y., LEBRÃO, M.L. \& JORGE, M.T. 1998. Óbitos por serpentes peçonhentas no estado de São Paulo: avaliação de 43 casos, 1988/1993. Rev. Ass. Med. Bras. 44(4):312-318.

SANTOS-FITA, D. \& COSTA-NETO, E.M. 2007. As interações entre os seres humanos e os animais: a contribuição da etnozoologia. Biotemas 20(4):99-10.

SILVA, M.V. 2006. Serpentes do Estado do Acre: riqueza, dieta, etnoconhecimento e acidentes ofídicos. Dissertação de Mestrado, Universidade Federal do Acre, Rio Branco.

TORRES, D.F., OLIVEIRA, E.S., ALVES, R.R.N. \& VASCONCELLOS, A. 2009. Etnobotânica e Etnozoologia em unidades de conservação: uso da biodiversidade na APA de Genipabu, Rio Grande do Norte, Brasil. Interciência 34(9):623-629.

UETZ., P. 2008. How many species? In TIGR reptile database (P. Uetz \& J. Hallerman) http://www.reptile-database.org/db-info/SpeciesStat.html (último acesso em 27/05/2010).

VIZOTTO, L.D. 2003. Serpentes: lendas, mitos, superstições e crendices. Plêiade, São Paulo. 
